\title{
Awareness, Attitudes, Practices, and Perceived Barriers to Medical Error Incident Reporting Among Faculty and Health Care Practitioners (HCPs) in a Dental Clinic
}

This article was published in the following Dove Press journal:

Journal of Multidisciplinary Healthcare

\section{Zainab Al-zain \\ Arwa Althumairi (D}

Department of Health Information Management and Technology, College of Public Health, Imam Abdulrahman Bin Faisal University, Dammam, Saudi Arabia
Correspondence: Arwa Althumairi Department of Health Information Management and Technology, College of

Public Health, Imam Abdulrahman Bin

Faisal University, P.O. Box 2954,

Dammam, 6603-342II, Saudi Arabia

Tel +966 I33335213

Email aalthumairi@iau.edu.sa
Background: Good Catch programs are being increasingly embraced by the Saudi healthcare system to improve incidence reporting rates and patient safety. However, dental health care is at a critical stage of promoting a safety culture; there is insufficient use of incident reporting systems (IRS) in several dental schools. Therefore, in this study, we evaluated the level of awareness, attitudes, practices, and perceived barriers to incident reporting among faculty and health care practitioners (HCPs) working in a university dental clinic.

Methods: It is a cross-sectional study design where participants have been recruited from faculty working in a dental clinic at Imam Abdulrahman bin Faisal University during the year 2019. A self-administered questionnaire was distributed including domains of awareness, attitude, practice, and barriers to use.

Results: A total of 199 completed questionnaires were received. HCPs differed with respect to their levels of awareness, attitudes, and practices of incident reporting when compared with non-Saudi HCPs. Nurses showed high awareness scores $(X=4.4, p<0.001)$, practice scores $(X=3.61, p<0.001)$, and attitudes $(X=3.9, p<0.001)$ in comparison with dentists and interns. The respondents agreed that the most common factor that influenced the rate of their incident reporting was "possible negative effect on the relationship with employees.".

Conclusion: Nurses showed higher levels of awareness, attitude, and practice regarding IRS than did dentists and interns. We uncovered key factors influencing incident reporting among the faculty and HCPs in a university dental clinic. These findings could aid policymakers to focus on these factors so as to frame appropriate strategies to encourage incident reporting and to improve the effective use of the IRS.

Keywords: incidence report, dental clinic, awareness, patient safety, healthcare providers

\section{Introduction}

Incidents in healthcare are unplanned undesirable events whose occurrence results in adverse events such as injury, a threat of malpractice claims, or hindrance of completion of a task. Incident reporting permits the appreciation of sentinel events, near misses, and potential malpractice threats, thereby facilitating the institution of suitable policies to prevent their recurrence. Several evidence-based investigations found low levels of reporting of healthcare incidents in Saudi Arabia., ${ }^{1,2}$ Consequently, underreporting of incidents contributes to poor patient safety outcomes such as increased incidence of medical errors. 
Understanding the factors underlying the underreporting of healthcare incidents may facilitate the recognition of critical corrective steps. Although there is a high level of awareness of incident reporting among healthcare providers, there are huge discrepancies among health workers' perceptions of incidents and reportable situations. Furthermore, many workers are unfamiliar with reporting policies and systems. ${ }^{3,4}$ Corrective interventions to enhance incident reporting should also appreciate that factors that discourage incident reporting vary among healthcare professionals. For example, while fear of blame is mentioned as a reason for underreporting by many physicians, this reason is less frequently mentioned by nurses. ${ }^{5}$ The systematic collection of data is an accompaniment to the enforcement of safety culture manifested by a blame-free environment, a focus on systematic improvement, and adequate leadership involvement in responding to incidents. ${ }^{6}$ Incident reporting systems are used as a means to streamline reporting in healthcare institutions.

Dental clinical settings are like other settings in the sense that they are bound to encounter challenges. This might cause deviation from implementing the correct processes; therefore, it is necessary to recognise the related risks to ensure that the best healthcare services are delivered. Especially in the Saudi Arabian context, various researchers have studied the healthcare professionals' knowledge, attitude, and practice towards medical error or adverse drug reactions reporting in Saudi tertiary care hospitals. ${ }^{5,7-9}$ A study revealed the barriers, facilitators, strategies, and predictors for adverse drug reactions reporting in three general hospitals in Saudi Arabia. ${ }^{10}$ Another study revealed the attitudes of dental professional staff and auxiliaries in Saudi dental institutions over the medical errors' disclosure. ${ }^{11}$ On reviewing these studies, no previous research has studied the knowledge, attitude, and practice towards incident reporting among faculty and health practitioners serving in the dental clinics of Saudi universities. To support this statement, we believe that the value of this study is its measure of the levels of awareness, practice, and attitudes of all faculty and health practitioners in the dental hospital towards incident reporting. There is an essential necessity for such studies that may promote the level of healthcare quality, especially in the culture of patient safety. This study also provides insight into the strengths and weaknesses of the incident reporting system. The findings of this study would help hospital administrators to frame the appropriate strategies to improve the knowledge, attitude and practice towards incident reporting among the healthcare professionals in a dental hospital setting.

\section{Methods}

\section{Study Design}

This study was a quantitative cross-sectional study of all faculty and health practitioners at Imam Abdulrahman bin Faisal University (IAU) who were recruited during 2019.

\section{Study Tool}

A self-administered questionnaire was distributed to the participants on site with a consent form statement.

The questionnaire was adapted from a published source, with no modifications. ${ }^{3}$ It contains several domains: awareness, attitude, practice, and barriers of incidence reporting. These items were designed to assess the perceptions of dental care practitioners towards incident reporting. The questionnaire included closed questions and was based on a five-point Likert scale from "Strongly Agree" to "Strongly Disagree" (Strongly Agree $=5$ to Strongly Disagree $=1$ ). Demographic details included age, gender, nationality, qualification, specialty, place of work, and years of experience. The level of agreement for the three domains (awareness, attitude, and practice) was calculated to give a total score of reporting level of agreement. Higher scores indicate more participant agreement with each domain.

Participants were asked several reasons on why they might not report an incidence, and the responding answers were coded as "Disagree," $3=$ "Neutral," or 4-5 = "Agree."

\section{Statistical Analysis}

A univariate analysis was performed to assess participant demographic characteristics (age, gender, nationality, years of experience, and speciality) in terms of numbers and percentages.

The normality of the sample was tested using calculation of skewness and kurtosis which were around -2.00 and 2.00. Then, the suitable bivariate analysis was conducted including the $t$-test for independent variables of less than one group such as gender and ANOVA for more than two groups at a level of significance of $95 \%$.

The analysis tested three objectives:

1. Identify the characteristics of the participant with awareness, attitude, and practice. 
2. Predict participant characteristics and frequency of incidence reporting and awareness and attitude.

3. Identification of common barriers of incidence reporting.

All statistical analyses were performed using SPSS version 27, Armonk, NY.

\section{Ethics and Limitations}

IRB approval was attained from the office of the Vice President for Research and Higher Studies, Imam Abdulrahman Bin Faisal University. The Institutional Review Board number was IRB -2020-PGS-03-014, approved on 27/01/2020. The data were aggregated, anonymized, and saved on a secure server.

All Participants were being willing to participate in the research program by providing an agreement on the paperbased questionnaire written consent statement, to agree to use their data for research purposes. Additionally, this study was conducted in accordance with the Declaration of Helsinki. The manuscript was designed to follow STROBE guidelines (Appendix 1).

\section{Results}

A total of 199 participants were included out of a total of $290(68.6 \%)$ employees who were in duty and receive the questioner during the study period. The majority were female, Saudi aged between 30 and 39 years old (Table 1); $25 \%$ were nurses, $24 \%$ were dentists and other specialties, and $50 \%$ had more than 5 years experience.

The level of awareness, practice, and attitude of the faculty and health practitioners towards medical error reporting concerning their demographic characteristics were described with the mean and standard deviation in Table 2. The median levels of awareness, practice, and attitude were similar between males and females. There was a statical significant difference between Saudi and non-Saudi in terms of median awareness scores, 3.9 and 4.1 , respectively $(\mathrm{p}=0.005)$. The type of specialty had a significant effect in terms of awareness, practice, and attitude. Nurses had higher awareness scores $(X=4.4$, $\mathrm{p}=<0.001)$, practice scores $(\mathrm{X}=3.44, \mathrm{p}=<0.001)$, and attitude scores $(X=4.1, p=<0.001)$ than did respondents in other specialties. Participants with less than 1 year of experience had higher scores of awareness ( $X=4.3$, $\mathrm{p}=0.082)$ than participants with more years of experience (Table 1).
Participants who had not reported an incident had lower awareness scores $(X=3.9$ vs $X=4.2, p=0.015)$ and practice scores $(\mathrm{X}=2.9$ vs $\mathrm{X}=3.75, \mathrm{p}=<0.001)$ than did participants who had reported an incident (Table 2).

There was differing agreement among participants in terms of the common factors that might influence the rate of reporting: $47 \%$ of participants disagreed with this statement "I do not want the case to be discussed" (Table 3). The most common factor that the participants agreed might influence the rate of reporting was "possible negative effect on the relationship with employees" with $20 \%$ in total agreement. This was followed by "I do not want the case to be discussed", "Management usually does not take action", and "Community is not supportive," with $14 \%$ each.

\section{Discussion}

In clinical settings, IRS has gained significance in terms of improvement of patient safety and delivering valuable information as to how and why the patients can be injured at the institutional level. There is increasing curiosity among hospital administrators regarding evaluations of safety of hospitals and health systems. The present study assessed the awareness, attitude, and practice towards IRS among faculty and HCPs working in Imam Abdulrahman bin Faisal university dental clinic. We also examined the perceived barriers to incident reporting. The results showed that the level of awareness, attitude, or practice of IRS among the faculty and HCPs of the IAU dental clinic did not differ with respect to the demographic characteristics except nationality and specialty. Besides, the relation between the participants' frequency of incidence reporting and their awareness, attitude, and practice on medical error reporting was revealed. The factors influencing incident reporting were found. Moreover, these findings were discussed with the appropriate literature as follows:

Concerning with awareness, we observed that there was no significant difference in terms of level of awareness towards IRS among faculty and health care practitioners (HCPs) with respect to gender, age, or working experience. Carandang et al observed that the gender of the health practitioners failed to significantly influence their knowledge, attitude, and practice towards medication error reporting. ${ }^{12}$ In that study, the authors observed that health practitioners in the middle-aged group (35-50 years old) showed the most knowledge towards medication error reporting. ${ }^{12}$ Concerning working experience, the findings 
Table I Bivariate Analysis Between Patient Characteristics and Median Score of Awareness, Practice, and Attitude

\begin{tabular}{|c|c|c|c|c|c|c|c|c|c|c|}
\hline \multirow{2}{*}{$\begin{array}{l}\text { Participant } \\
\text { Characteristics }\end{array}$} & \multirow{2}{*}{$\begin{array}{c}N(\%) \\
N=199\end{array}$} & \multicolumn{3}{|c|}{ Median Awareness Score } & \multicolumn{3}{|c|}{ Median Practice Score } & \multicolumn{3}{|c|}{ Median Attitude Score } \\
\hline & & $\begin{array}{l}\text { Mean } \\
(\mathrm{SD})\end{array}$ & t-Value & p-value & $\begin{array}{l}\text { Mean } \\
(\mathrm{SD})\end{array}$ & t-Value & p-value & $\begin{array}{l}\text { Mean } \\
(\mathrm{SD})\end{array}$ & t-Value & p-value \\
\hline \multicolumn{11}{|l|}{ Gender } \\
\hline Male & $62(31)$ & $4.09(0.70)$ & 0.338 & 0.736 & $3.27(1.16)$ & -0.740 & 0.557 & $3.83(0.76)$ & -0.325 & 0.325 \\
\hline Female & $137(69)$ & $4.036(0.77)$ & & & $3.29(1.09)$ & & & $3.84(0.95)$ & & \\
\hline \multicolumn{11}{|l|}{ Nationality } \\
\hline Saudi & $115(58)$ & $3.90(0.85)$ & -0.618 & $0.005^{*}$ & $3.03(1.19)$ & -0.391 & $0.001 *$ & $2.91(9.11)$ & 1.221 & 0.115 \\
\hline Non-Saudi & $84(42)$ & $4.21(0.59)$ & & & $3.63(0.89)$ & & & $3.73(0.76)$ & & \\
\hline \multicolumn{11}{|l|}{ Age (years) } \\
\hline$<30$ & $66(33)$ & $4.12(0.78)$ & 0.368 & 0.693 & $3.38(1.11)$ & 0.569 & 0.558 & $4.02(0.91)$ & 2.010 & 0.138 \\
\hline $30-39$ & $91(45)$ & $4.00(0.77)$ & & & $3.19(1.11)$ & & & $3.69(0.80)$ & & \\
\hline$>39$ & $42(2 I)$ & $4.03(0.72)$ & & & $3.33(1.16)$ & & & $3.84(0.85)$ & & \\
\hline \multicolumn{11}{|l|}{ Speciality } \\
\hline Dentist & $48(24)$ & $3.21(0.56)$ & 18.70 & $<0.00 I^{*}$ & $2.54(1.07)$ & 12.510 & $<0.00 I^{*}$ & $3.27(0.67)$ & 5.641 & $0.00 I^{*}$ \\
\hline Nurse & $51(25)$ & $4.4(0.49)$ & & & $3.62(0.98)$ & & & $3.94(0.77)$ & & \\
\hline Intern & $18(9)$ & $4.25(0.62)$ & & & 3.44 (1.09) & & & $4.08(0.86)$ & & \\
\hline Student & $35(18)$ & $3.96(0.87)$ & & & 2.97 (1.09) & & & $3.96(0.99)$ & & \\
\hline Other & $47(24)$ & $4.33(0.59)$ & & & $3.83(0.84)$ & & & $4.14(0.76)$ & & \\
\hline \multicolumn{11}{|l|}{ Experience } \\
\hline I year and less & $37(19)$ & $4.28(0.58)$ & 2.260 & 0.082 & $3.44(1.05)$ & 2.055 & 0.108 & $3.87(0.8 \mathrm{I})$ & 1.060 & 0.369 \\
\hline $2-5$ years & $60(30)$ & $4.00(0.78)$ & & & $3.27(1.12)$ & & & $3.85(0.91)$ & & \\
\hline More than 5 years & $100(50)$ & $3.94(0.79)$ & & & $3.19(1.12)$ & & & $3.78(0.85)$ & & \\
\hline Prefer not to say & $3(2)$ & $4.67(0.58)$ & & & $4.67(0.58)$ & & & $4.67(0.86)$ & & \\
\hline
\end{tabular}

Note: *P-value is $<0.001$.

Table 2 Relation Between Awareness, Practice and Attitude Median Score and Frequency of Incidence Reporting

\begin{tabular}{|c|c|c|c|c|c|c|c|c|c|c|}
\hline \multirow{2}{*}{$\begin{array}{l}\text { Participant } \\
\text { Characteristics }\end{array}$} & \multirow{2}{*}{$\begin{array}{c}N(\%) \\
N=199\end{array}$} & \multicolumn{3}{|c|}{ Median Awareness Score } & \multicolumn{3}{|c|}{ Median Practice Score } & \multicolumn{3}{|c|}{ Median Attitude Score } \\
\hline & & Mean (SD) & t-Value & p-value & Mean (SD) & t-Value & p-value & Mean (SD) & t-Value & p-value \\
\hline \multicolumn{11}{|c|}{$\begin{array}{l}\text { Encountered but not } \\
\text { reported last months? }\end{array}$} \\
\hline No & $115(58)$ & $4.02(0.77)$ & -0.460 & 0.619 & 3.25 (1.14) & -0.547 & 0.547 & $3.91(0.93)$ & 1.111 & 0.268 \\
\hline Yes & $84(42)$ & $4.08(0.74)$ & & & $3.33(1.08)$ & & & $3.74(0.76)$ & & \\
\hline \multicolumn{11}{|c|}{$\begin{array}{l}\text { Reported incidents in } \\
\text { writing last year? }\end{array}$} \\
\hline No & III (56) & $3.90(0.8 \mathrm{I})$ & -2.451 & $0.015^{*}$ & $2.91(1.10)$ & -5.560 & $<0.001 *$ & $3.87(0.88)$ & -0.548 & 0.585 \\
\hline Yes & $87(44)$ & $4.20(0.66)$ & & & $3.75(0.96)$ & & & $3.89(0.83)$ & & \\
\hline
\end{tabular}

Note: ${ }^{*}$-value is $<0.001$.

of our study are in line with those of previous studies that found that there was no significant difference in medication error reporting behaviour among the various groups of health practitioners in terms of the years of experience or unit of practice. $^{12-14}$

Regarding nationality, non-Saudi faculty and HCPs were more aware of IRS than were Saudis, although it was statically significant the difference in score is very close only 0.2 difference, they all fell under high awareness level. There was a significant difference in the level of awareness towards IRS among the specialty of the faculty and HCPs. In particular, nurses reported a higher level of awareness towards IRS than did other specialties, which is in accord with the findings of Alsulami et al, who 
Table 3 Distribution of Number of Participants “Agree," “Neutral," and "Disagree” per Factor Influence of Incidence Reporting

\begin{tabular}{|c|c|c|c|c|c|c|}
\hline \multirow[t]{2}{*}{ Factors } & \multicolumn{2}{|c|}{ Agree } & \multicolumn{2}{|c|}{ Neutral } & \multicolumn{2}{|c|}{ Disagree } \\
\hline & $\mathbf{N}$ & $\%$ & $\mathbf{N}$ & $\%$ & $\mathbf{N}$ & $\%$ \\
\hline I do not want to be held responsible & 78 & 39 & 74 & 37 & 47 & 24 \\
\hline I do not think that employee should go through a disciplinary actions & 89 & 45 & 44 & 22 & 66 & 33 \\
\hline I do not want the case to be discussed & 47 & 24 & 63 & 32 & 89 & 45 \\
\hline The complexity of the incident reporting form & 65 & 33 & 71 & 36 & 63 & 32 \\
\hline Community is not supportive & 97 & 49 & 53 & 27 & 49 & 25 \\
\hline Confidentiality is not guaranteed & 71 & 36 & 41 & 21 & 87 & 44 \\
\hline Management usually does not take action & 92 & 46 & 52 & 26 & 55 & 28 \\
\hline Possible negative effect on the relationship with employees & 132 & 66 & 38 & 19 & 29 & 15 \\
\hline
\end{tabular}

observed that the nurses demonstrated higher reporting knowledge than did their peers. ${ }^{5}$ By contrast, Km, B et al observed that pharmacists had more knowledge of adverse drug reaction (ADR) reporting than did nurses or other specialties. ${ }^{15}$

While reviewing the attitude scores, faculty and HCPs did not differ with respect to gender, nationality, age, or working experience. Similarly, Carandang et al reported that factors such as age, gender, and hospital experience did not affect health practitioners' attitudes. ${ }^{12}$ By contrast, Alsulami reported that non-Saudis nurses showed higher favourable attitudes towards medication error reporting than did Saudis. ${ }^{4}$

On the other hand, there was a significant difference in attitude towards IRS among the various specialties of the faculty and HCPs. The "others" category, followed by interns, showed higher attitude scores than the remaining categories of specialty. Unlike our result, Khan observed no significant variation in attitude towards ADR reporting among different positions of doctors. ${ }^{16}$ Kapil and Anoopjit reported that staff nurses showed an average positive attitude towards incident reporting in a tertiary hospital setting. ${ }^{17}$

Regarding the practice, there were significant differences in IRS practice among the faculty and HCPs with respect to nationality and specialty. Non-Saudis had higher practice scores than Saudis. The "others" category, followed by nurses, reported higher practice scores than the remaining categories. Contrary to these findings, Alblaihad et al found that Saudis frequently reported incidents verbally to their organisation and completed incident forms. ${ }^{3}$ They also showed a higher frequency rate of reporting incidents than did the others. Saudis showed higher positive practice scores than did non-Saudis, and nurses had higher rates of reporting incidents than did others. Previous studies conducted in Malaysia demonstrated poor practice among physicians. ${ }^{18-20}$ By contrast, Carandang et al reported no significant variation in the practice of HCPs such as nurses, pharmacists, and physicians. $^{12}$

We found no significant differences in IRS practice among the faculty and HCPs with respect to gender, age, or working experience. In accordance with these results, Carandang et al found that the age and gender of HCPs failed to strongly impact medication error reporting. ${ }^{12}$ Previous studies found that the health practitioners' practices regarding medication error reporting had no significant associations with years of clinical experience and were not significantly associated with years of hospital experience. $^{12,14}$ Although this had almost all faculty in the hospital, it was conducted in one educational nonprofit healthcare settings, therefore the generalizability of this study could be taken in caution.

These findings summarize that the faculty and health practitioners of a Saudi university dental clinic did not vary in the level of awareness, attitude, and practice concerning all demographic characteristics except nationality and speciality. This might be due to the equal opportunity of the exposure of those participants to the training programs on IRS; however, the difference in their nationality and speciality might be due to their varied perception with the educational background and race.

Besides, we found no significant differences in awareness, practice, or attitude score between the faculty and HCPs who responded "Yes" and "No" to incidents encountered but not reported in the previous months. We found that $58 \%$ of the faculty and HCPs encountered incidents and reported them in the previous months. In accord with this result, Gidey et al observed that $74.9 \%$ of HCPs encountered ADRs in the previous 12 months of their clinical practice. ${ }^{21} \mathrm{We}$ found that $56 \%$ of the faculty 
and HCPs failed to report incidents in writing in the previous year. This finding is similar to that of Gidey et al, who reported that $68 \%$ of HCPs did not report ADRs, even when they encountered them. ${ }^{21}$

On analysing the factors influencing the incident reporting, we found that $66 \%$ of the faculty and HCPs agreed that the "possible negative effect on the relationship with employees" was the most common factor influencing incident reporting. Khan observed that only $10 \%$ of HCPs perceived the "lack of confidence to discuss an ADR with a colleague" as a factor discouraging ADR reporting. ${ }^{16}$ In the present study, we observed that about $45 \%$ of the faculty and HCPs disagreed that the item "I do not want the case to be discussed" influenced their incident reporting. Contrary to this finding, Alblaihed et al observed that $21 \%$ of dental clinical supervisors rated "I do not want the case to be discussed" as a barrier to incident reporting. ${ }^{3}$

There were some limitations on the study design as it was a retrospective cross-sectional study design based on a questionnaire, some information such as the number of reported incidences might be subject to recall bias and were not able to detect the incidence while it is happening. However, the study was population-based and was designed to assess their practice during a one-year period that the memorization of incidence within this period might be still reliable. It is suggested to conduct a longitudinal study design to monitor the progress and awareness of incidence reporting in the current institutions for optimal patient safety measurement.

\section{Conclusion}

We found no significant differences in the awareness, attitude, or practice of IRS among the faculty and HCPs of the IAU dental clinic concerning all demographic characteristics except nationality and specialty. Further, NonSaudi faculty and HCPs were more highly aware of IRS and practicing it than Saudis. However, faculty and HCPs showed an equal attitude towards IRS concerning their nationality. A significant difference in the awareness, attitude, or practice of IRS among the respondents' specialty. Besides, there is room for improving the practice of reporting IRS among the faculty and HCPs. The most common factor influencing incident reporting was observed as the possibility of a negative effect on employees' relationships. This study is only limited to a Saudi university dental clinic. To generalize these findings, further research is warranted to cover the healthcare professionals working in the dental clinic of all Saudi universities.

\section{Disclosure}

The authors report no conflicts of interest for this work.

\section{References}

1. Ali R. Barriers to sentinel events reporting in Tertiary Hospital at Dammam, Saudi Arabia. Open Sci J. 2018;3(4). doi:10.23954/osj. v3i4.1532

2. Alsafi E, Baharoon S, Ahmed A, Al Jahdali H, Al Zahrani S, Al Sayyari A. Physicians' knowledge and practice towards medical error reporting: a cross-sectional hospital-based study in Saudi Arabia. East Mediterr Health J. 2015;21(9):655-664. doi:10.26719/2015.21. 9.655

3. AlBlaihed RM, AlSaeed MI, Abuabat AA, Ahsan SH. Incident reporting in dentistry: clinical supervisor's awareness, practice and perceived barriers. Eur J Dent Educ. 2018;22(3):e408-18. doi:10. 1111/eje. 12319

4. Banakhar M, Tambosi A, Asiri S, Banjar Y, Essa Y. Barriers of reporting errors among nurses in a Tertiary Hospital. Int $J$ Nurs Clin Pract. 2017;4(1):1-7. doi:10.15344/2394-4978/2017/245

5. Alsulami SL, Sardidi HO, Almuzaini RS, et al. Knowledge, attitude and practice on medication error reporting among health practitioners in a tertiary care setting in Saudi Arabia. Saudi Med J. 2019;40 (3):246-251. doi:10.15537/smj.2019.3.23960

6. Alswat K, Abdalla RAM, Titi MA, et al. Improving patient safety culture in Saudi Arabia (2012-2015): trending, improvement and benchmarking. BMC Health Serv Res. 2017;17(1):516. doi:10.1186/ s12913-017-2461-3

7. Abdel-Latif MM. Knowledge of healthcare professionals about medication errors in hospitals. J Basic Clin Pharm. 2016;7(3):87. doi:10.4103/0976-0105.183264

8. AlShammari TM, Almoslem MJ. Knowledge, attitudes \& practices of healthcare professionals in hospitals towards the reporting of adverse drug reactions in Saudi Arabia: a multi-centre cross sectional study. Saudi Pharm J. 2018;26(7):925-931. doi:10.1016/j.jsps.2018.04.012

9. Alsafi E, Baharoon S, Ahmed A, Al-Jahdali HH, Al Zahrani S, Al Sayyari A. Physicians' knowledge and practice towards medical error reporting: a cross-sectional hospital-based study in Saudi ArabiaEast Mediterr Health J. 2015;21(9):655-664. doi:10.26719/2015.21.9.655.

10. Bakhsh TMA, Al-Ghamdi MS, Bawazir SA, Qureshi NA. Barriers, facilitators, strategies, and predictors for reporting adverse drug reactions in three general hospitals in Jeddah, 2013. J Adv Med Med Res. 2016;1-13.

11. Al-Nomay NS, Ashi A, Al-Hargan A, Alshalhoub A, Masuadi E. Attitudes of dental professional staff and auxiliaries in Riyadh, Saudi Arabia, toward disclosure of medical errors. Saudi Dent J. 2017;29 (2):59-65. doi:10.1016/j.sdentj.2017.01.003

12. Carandang RR, Resuello D, Hocson G, Respicio K, Reynoso C. Knowledge, attitude and practices on medication error reporting among health practitioners from Hospitals in Manila. SAS J. 2015;4:293-300.

13. Mayo AM, Duncan D. Nurse perceptions of medication errors: what we need to know for patient safety. J Nurs Care Qual. 2004;19 (3):209-217. doi:10.1097/00001786-200407000-00007

14. Armutlu M, Foley M-L, Surette J, Belzile É, McCusker J. Survey of nursing perceptions of medication administration practices, perceived sources of errors and reporting behaviours. Healthc Q. 2008;11 (3):58-65.

15. Km B, Reddy BS Assessment of knowledge, attitude and perception of healthcare professionals towards adverse drug reactions reporting: a Questionnaire Based Survey. 2017;3:9. 
16. Khan SA, Goyal C, Tonpay SD. A study of knowledge, attitudes, and practice of dental doctors about adverse drug reaction reporting in a teaching hospital in India. Perspect Clin Res. 2015;6(3):144-149. doi:10.4103/2229-3485.159938

17. Kapil S, Anoopjit K. A study to assess the knowledge, attitude and perceived barriers on incident reporting among staff nurses working in a Tertiary Care Hospital, Ludhiana, Punjab. IJONE. 2020;12(1):142-145.

18. Agarwal R, Daher AM, Mohd Ismail N. Knowledge, practices and attitudes towards adverse drug reaction reporting by private practitioners from Klang Valley in Malaysia. Malays J Med Sci. 2013;20 (2):52-61.

19. Mohamed IN, Borhanuddin B, Shuid AN, Fozi NFM. Attitudes, perception and knowledge of general practitioners towards adverse drug reaction (ADR) reporting in Malaysia-A pilot study. Saudi Pharm J. 2013;1(1).
20. Abubakar AR, Simbak NB, Haque M. A systematic review of knowledge, attitude and practice on adverse drug reactions and pharmacovigilance among doctors. J App Pharm Sci. 2014;4(10):117-127. doi:10.7324/JAPS.2014.401021.

21. Gidey K, Seifu M, Hailu BY, Asgedom SW, Niriayo YL. Healthcare professionals knowledge, attitude and practice of adverse drug reactions reporting in Ethiopia: a cross-sectional study. BMJ Open. 2020;10(2):e034553. doi:10.1136/bmjopen-2019-034553.
Journal of Multidisciplinary Healthcare

\section{Publish your work in this journal}

The Journal of Multidisciplinary Healthcare is an international, peerreviewed open-access journal that aims to represent and publish research in healthcare areas delivered by practitioners of different disciplines. This includes studies and reviews conducted by multidisciplinary teams as well as research which evaluates the results or conduct of such teams or healthcare processes in general. The journal

\section{Dovepress}

covers a very wide range of areas and welcomes submissions from practitioners at all levels, from all over the world. The manuscript management system is completely online and includes a very quick and fair peer-review system. Visit http://www.dovepress.com/testimonials. php to read real quotes from published authors. 